\title{
Estructura, composición florística y almacenamiento de carbono en bosques nativos del páramo de Anaime, Tolima, Colombia
}

\author{
Structure, floristic composition and carbon storage in native forests of paramo de Anaime, \\ Tolima, Colombia
}

Milena Andrea Segura Madrigal ${ }^{I}$, Hernán Jair Andrade Castañeda", Carlos Arturo Mojica Sánchez ${ }^{\text {III }}$

\section{Resumen}

Los páramos tienen alta importancia ambiental por su alto endemismo, su regulación hídrica y su almacenamiento de carbono. En este trabajo se caracterizó la estructura y composición poblacional de la vegetación arbórea, se estimó el almacenamiento de carbono en biomasa aérea y el potencial de reducción de emisiones por deforestación, y se priorizaron los tipos de bosques nativos en los esfuerzos de conservación en la Reserva Natural Semillas de Agua (RNSA) en el páramo de Anaime, Tolima, Colombia. Se realizó un muestreo aleatorio estratificado, en donde cada tipo de bosque (achaparrado, maduro y subpáramo) es un estrato. En cada tipo de bosque, se establecieron nueve parcelas de muestreo de $10 \times 15 \mathrm{~m}$, donde se midieron todos los individuos con un diámetro del tronco a la altura del pecho (dap) $\geq 10 \mathrm{~cm}$. La biomasa aérea se estimó a través de un modelo alométrico multi-especie generado localmente. Las especies importantes, en términos de índice de valor de importancia y de almacenamiento de carbono, fueron Weinmannia auriculata y Miconia spp. El mayor almacenamiento de carbono se alcanzó en los bosques del subpáramo, seguido de los maduros y achaparrados (242 vs 147 vs $25 \mathrm{Mg} / \mathrm{ha}$, respectivamente). Los bosques en la RNSA almacenan en total 3,2 $\mathrm{Tg} \mathrm{CO}_{2}$ e en biomasa aérea. Sin embargo, se podrían perder 0,2-0,5 $\mathrm{Tg}$ $\mathrm{CO}_{2}$ e en 20 años con una deforestación anual de 0,78 y $0,28 \%$, respectivamente. Estas reducciones de gases de efecto invernadero podrían ser comercializadas en un potencial proyecto REDD+, que ayuden a conservar estas áreas de bosque. La conservación de estos bosques es clave para evitar grandes emisiones de $\mathrm{CO}_{2}$. Los bosques achaparrados y del subpáramo deberían ser priorizados en los esfuerzos de conservación debido a su diversidad, endemismo y a su gran cantidad de carbono en el caso de los bosques maduros.

Palabras clave: Bosque alto andino; Deforestación; Índice de valor de importancia; Reducción de emisiones

\begin{abstract}
Páramos have a high environmental importance due to their high plant and animal endemism, water regulation and carbon storage. In this work, the structure and composition of the tree vegetation were characterized as well as carbon storage in aboveground biomass and the potential reduction of emissions by deforestation were estimated prioritizing the type of forest for conservation efforts in the Reserva Natural Semillas de Agua (RNSA) in Páramo of Anaime, Tolima, Colombia. A stratified randomly sampling, where type of forests (stumpy, mature and subpáramo forests) was the strata, was used. In each type of forest, a total of nine sampling plots with an area of $10 \times 15 \mathrm{~m}$ were established to measure all individuals with diameter at breast height $(\mathrm{dbh}) \geq 10 \mathrm{~cm}$. Total aboveground was estimated using a multi-species allometric model locally generated. The most important trees species, in terms of the importance value index and carbon storage, were Weinmannia auriculata and Miconia spp. The greatest carbon storage was reached by the subpáramo forests, followed by mature and stumpy forests ( 242 vs $147 \mathrm{vs} 25 \mathrm{Mg} / \mathrm{ha}$, respectively). The RNSA forests stored a total of $3.2 \mathrm{Tg} \mathrm{CO}_{2} \mathrm{e}$ in the total aboveground biomass. However, 0.2-0.5 $\mathrm{Tg} \mathrm{CO}_{2 \mathrm{e}}$ can be lost in 20 years with an annual deforestation of 0.78 or $2.8 \%$, respectively. These reductions of greenhouse gases could be commercialized in a REDD+ potential project that helps to conserve these forest areas. The conservation of these forests is a key for avoiding the emission of high amounts of $\mathrm{CO}_{2}$. The stumpy and subpáramo forests should be prioritized in the conservation efforts due to their high diversity and endemism and the high amount of carbon in the case of mature forests.
\end{abstract}

Keywords: High-land forests; Deforestation; Importance value index; Reduction of emissions

\footnotetext{
I Ingeniera Forestal, MSc., Facultad de Ingeniería Forestal, Grupo de Investigación PROECUT, Universidad del Tolima, Barrio Santa Helena Parte Alta, CP 730008, Ibagué (Tolima), Colombia. masegura@ut.edu.co (ORCID: 0000-0002-4813-1224)

II Ingeniero Agrónomo, PhD., Facultad de Ingeniería Agronómica, Grupo de Investigación PROECUT, Universidad del Tolima, Barrio Santa Helena, Parte Alta, CP 730008, Ibagué (Tolima), Colombia. hjandrade@ut.edu.co (ORCID: 0000-0002-3398-294X)

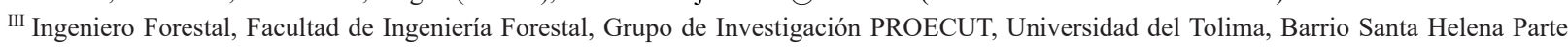
Alta, CP 730008, Ibagué (Tolima), Colombia. camojica@ut.edu.co (ORCID: 0000-0002-5396-7421)
} 


\section{Introducción}

Los bosques son una importante alternativa para mitigar las emisiones de gases de efecto invernadero (GEI), especialmente el $\mathrm{CO}_{2}$, principal causa del cambio climático, por lo cual los proyectos de reducción de emisiones por deforestación y degradación (REDD+) están tomando gran importancia. En esta estrategia, los bosques nativos son un uso del suelo clave (COOMES et al., 2008), ya que además de almacenar carbono, ayudan a la protección hídrica, la conservación de la diversidad y brindan belleza escénica (BISHOP y LANDELL-MILLS, 2003), lo cual representa beneficios adicionales para este tipo de proyectos. Aunque Colombia aún no tiene una estrategia finalizada para REDD+, entre 2009 y 2012 más de U\$33 millones han sido comprometidos, de los cuales U\$15,2 millones se han desembolsado (GÓMEZ et al., 2013).

Según FAO (2010), los bosques del mundo almacenan 289 Pg C en su biomasa, pero se ha presentado una reducción de estas reservas a un ritmo de 0,84 y 0,34 Pg C/año entre 1990 y 2000 y entre 2010 y 2015 , respectivamente (KÖHL et al., 2015). Estos cambios se deben principalmente a una reducción en el área de bosques; por ejemplo, América del Sur experimentó las mayores pérdidas netas de bosque en la primera década del siglo XXI (4 millones de hectáreas por año), lo cual le acarreó una pérdida total de cerca de 8,3 Pg C entre 1990 y 2015. En las últimas décadas, la deforestación ha sido una de las principales amenazas de los bosques del trópico colombiano (FAO, 2010), alcanzando una pérdida de cerca del $10 \%$ de sus bosques en los últimos 20 años (MADS, 2012). La situación de los bosques andinos es aún más crítica, con una pérdida del 39\% entre 1985 y 2000 (RUDAS et al., 2007). El páramo de Anaime, sitio del presente estudio, tuvo una tasa anual de deforestación $0,28 \%$ entre 2000 y 2010 (CORPORACIÓN SEMILLAS DE AGUA \& WWF COLOMBIA, 2011), con una disminución actual de este fenómeno, lo cual no implica que el riesgo de cambio de uso del suelo hacia actividades agropecuarias no está todavía latente.

Los páramos se encuentran exclusivamente en el trópico a altitudes superiores a los $3000 \mathrm{~m}$ (VÁSQUEZ y BUITRAGO, 2011) y son muy importantes para mitigar el cambio climático, pero a su vez son muy vulnerables a este fenómeno. Los bosques achaparrados corresponden a bosques enanos o de transición que se caracterizan por una vegetación achaparrada, muy densa y bastante diversa con plantas de tallos retorcidos o inclinados, de madera fuerte, follaje coriáceo, muchas veces con abundante pubescencia, lámina convexa y de márgenes enrolladas, hojas pequeñas y muy coriáceas. El bosque maduro presenta árboles con una altura de 8-10 m con diámetros promedios de árboles de $30 \mathrm{~cm}$ de dap en donde dominan las Ericáceas, encenillos (Weinmannia spp.), silba silba (Hedyosmun sp.) y nigüitos (Miconia spp.). En el bosque del subpáramo presenta una vegetación dominada por árboles, arbustos y matorrales (Diplostephium, Hesperomeles y Vaccinium) y un estrato de briofitas muy abundantes (ASORED, 2006).

Fehse et al. (2002) y Spracklen y Righelato (2014) afirman que los bosques de altura pueden capturar grandes cantidades de carbono en su biomasa, tal como aquellos con especies del género Polylepis (hasta 2 $\mathrm{Mg}$ C/ha/año), lo cual es superior a lo reportado por Gillespie et al. (1992), Lugo y Brown (1992), Segura et al. (2000), Salimon et al. (2011) y Köhl et al. (2015) en bosques tropicales. El cambio climático afecta estos ecosistemas, tanto en su diversidad florística como en su capacidad de captura de carbono, lo cual está siendo afectado por el manejo, tal como la intensificación de la ganadería (ANDRADE et al., 2014). Las especies de anfibios y reptiles, comúnmente empleadas como indicadores de biodiversidad, más vulnerables al cambio climático en Colombia son las que habitan ambientes andinos (páramos) y que presentan rangos altitudinales estrechos (URBINA-CARDONA, 2011). Los bosques de altura se reconocen como ecosistemas de alto endemismo (KESSLER, 2002) y diversidad florística, encontrándose más diversidad en la Cordillera Oriental del país (RANGEL-CHURIO y PINTO-ZÁRATE, 2012). En el Parque Nacional Podocarpus de Ecuador, se encontró un total de 412 especies de plantas vasculares, siendo 52 de éstas endémicas (LOZANO et al., 2010).

Medellín (2011) confirma la importancia de contar con métodos para valorar económicamente los bienes y servicios ambientales que los páramos proveen. La información sobre la capacidad de mitigación del cambio climático por ecosistemas boscosos en los páramos es fundamental para plantear políticas que permitan su protección, tal como proyectos de reducción o remoción de emisiones de GEI (REDD+) (RÜGNITZ et al. 2009). Sin embargo, hay muy poca información sobre este tema en bosques montanos y el número de estudios se reduce con la altitud (FEHSE et al., 2002).

El objetivo de este estudio es caracterizar la estructura y composición poblacional de la vegetación arbórea, estimar el almacenamiento de carbono en biomasa aérea y el potencial de reducción de emisiones por deforestación en el páramo de Anaime, Tolima, Colombia. 


\section{Materiales y métodos}

El presente estudio se realizó en la Reserva Natural Semillas de Agua (RNSA) y sus alrededores, localizada en el páramo de Anaime en el centro del departamento del Tolima, Colombia (Figura 1) y que pertenece a la Corporación Semillas de Agua, Organización No Gubernamental (ONG) de carácter nacional (CORPORACIÓN SEMILLAS DE AGUA, 2009). La reserva se encuentra en la Cordillera Central a una altitud entre 3200 y $3900 \mathrm{~m}$ y se caracteriza por tener terrenos abruptos con fuertes pendientes. Se proyectó incrementar el área de la reserva hasta las 15000 ha, de las cuales un $62 \%$ son bosques nativos y el restante corresponde mayoritariamente a pasturas. La RNSA se encuentra en la zona de vida de bosque muy húmedo montano (HOLDRIDGE, 1967), y tiene una precipitación media anual de 1700-1850 mm, humedad relativa promedio de $85 \%$ y temperatura de 3,0 a $18,7^{\circ} \mathrm{C}$ (CORPORACIÓN SEMILLAS DE AGUA, 2009) y posee suelos derivados de cenizas volcánicas, los cuales son utilizados en ganadería intensiva (ASORED, 2006).

Se seleccionaron los tres tipos de bosques más dominantes de la RNSA: achaparrado, maduro y subpáramo. Con ayuda de cartografía y mapas, se escogieron los sitios en donde se establecieron las parcelas de muestreo cubriendo toda el área de estudio en un muestreo aleatorio estratificado, donde los estratos son los tres tipos de bosques. El bosque maduro (3200 - $3500 \mathrm{~m}$ de altitud), con un área aproximada de 5370 ha, es el de mayor cobertura en la RNSA, seguido por los achaparrados ( $>3500 \mathrm{~m})$ y del subpáramo $(<3200 \mathrm{~m})$ con 2589 y 1291,4 ha, respectivamente.

\section{Figura 1 - Ubicación de las parcelas de muestreo en el Páramo de Anaime, Tolima, Colombia.}

Figure 1- Location of sampling plots in Páramo de Anaime, Tolima, Colombia.

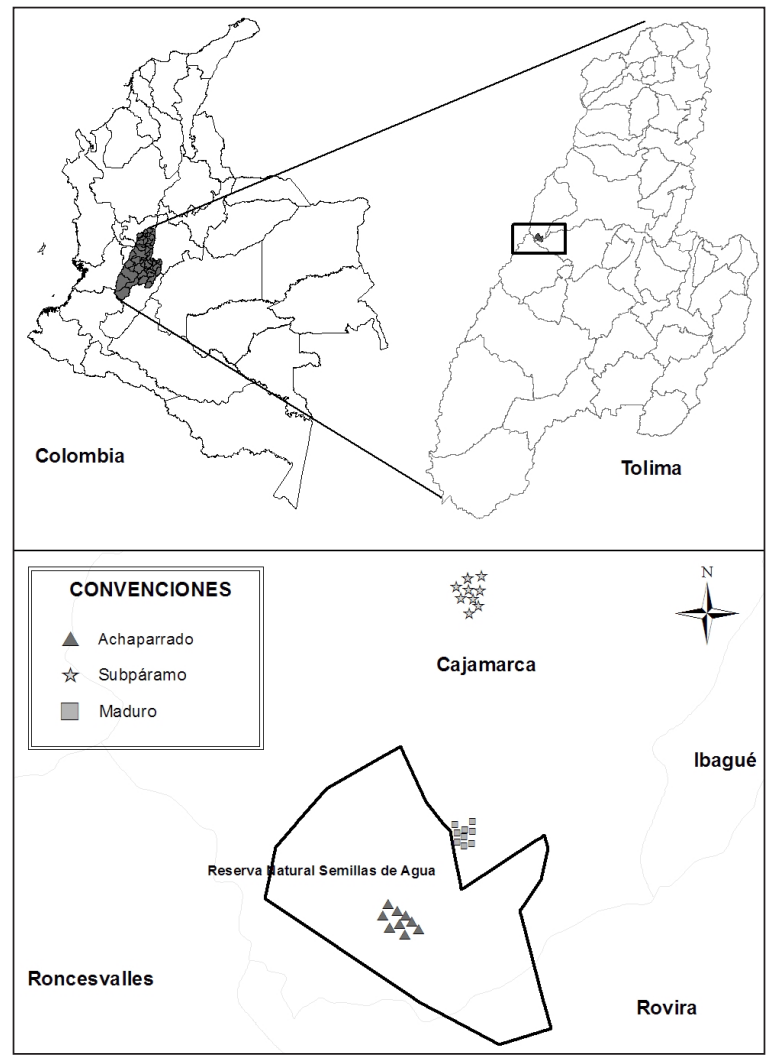

Se establecieron aleatoriamente nueve parcelas de muestreo (cinco temporales y cuatro permanentes) de $10 \times 15 \mathrm{~m}$ por tipo de bosque, las cuales se consideraron como repeticiones, lo que concuerda con los $25 \mathrm{~m}^{2}$ recomendados por Peyre (2015) en páramos y super-páramos. La mayor área de las parcelas de este estudio se debe a que se involucraron otro tipo de bosques con vegetación de mayores dimensiones. En cada parcela, se midió el diámetro del tronco a la altura del pecho (dap) y la altura total de todas las especies leñosas perennes con un dap $\geq 10 \mathrm{~cm}$. Se identificaron los individuos, en su mayoría a nivel de especie y/o género, con la experiencia 
de pobladores locales y la recolección de muestras para su identificación en el Herbario Toli de la Universidad del Tolima. Las familias a las cuales pertenecen las especies y/o géneros junto con sus identificadores fueron consultadas de la base de datos de The Plant List de países andinos (Bolivia, Ecuador y Perú).

Se estimó el valor absoluto y relativo de la abundancia, dominancia en términos de área basal y frecuencia para estimar el índice de valor de importancia (IVI). Adicionalmente se estimó el índice de Margalef, el índice de dominancia de Simpson y de equidad de Shannon-Wiener para evaluar la diversidad alfa, y los coeficientes cualitativos de similitud de Jaccard y cuantitativo de similitud de Sørensen además de la complementariedad para valorar la diversidad beta.

Se utilizó un modelo alométrico local (Ecuación 1) para estimar la biomasa en función del dap, el cual incluye las especies más dominantes de estos bosques con un dap entre 5 y $60 \mathrm{~cm}$ y una altura total de 4,2 a 25,5 m (SEGURA et al., 2013).

$$
\operatorname{Ln}(B)=-1,85+2,11 * \operatorname{Ln}(\text { dap }) \quad R^{2}=0,94 \quad \text { Ecuación } 1
$$

Donde: $\mathrm{B}=$ biomasa aérea $(\mathrm{kg} /$ árbol); dap = diámetro del tronco a la altura del pecho $(\mathrm{cm})$.

El carbono almacenado en la biomasa aérea fue estimado al multiplicar esta última por una fracción de 0,5 (BROWN y LUGO, 1982; IPCC, 2006). El almacenamiento total de carbono en la biomasa aérea para la RNSA fue estimado considerando el promedio del almacenamiento y el área total de cada tipo de bosque. El carbono fue convertido a $\mathrm{CO}_{2}$ multiplicando por 3,67, de acuerdo a su equivalencia estequiométrica (IPCC, 2006).

Se estimó el potencial de reducción de emisiones de GEI por efecto de la reducción de la deforestación en la RNSA durante un periodo de 20 años, tal como lo recomiendan Angelsen et al. (2009) al usar el Tier 1 (Nivel 1). La línea base se asumió como el cambio en el almacenamiento de carbono al aplicar las dos tasas de deforestación de 0,28 y $0,78 \%$ /año, correspondientes a la pérdida de bosques del páramo de Anaime entre 2000 y 2010 (CORPORACIÓN SEMILLAS DE AGUA \& WWF COLOMBIA, 2011) y de Colombia entre 2005 y 2010 (CABRERA et al., 2011), respectivamente. La situación con proyecto se consideró como aquella en la que se mantiene el carbono en la biomasa aérea al eliminar totalmente la deforestación durante el periodo de simulación. El almacenamiento de carbono aéreo total en la RNSA se estimó anualmente tomando cada tasa de deforestación y empleando la siguiente ecuación:

$$
C_{i+1}=C_{i} *\left[\frac{100-t}{100}\right] \quad \text { Ecuación } 2
$$

Donde: $C_{i+1}=$ almacenamiento de carbono en el año $i+1(\mathrm{Gg}) ; C_{i}=$ almacenamiento de carbono en el año $i(\mathrm{Gg}) ; t=$ tasa de deforestación (\%/año).

Los datos de abundancia, dap, altura total, área basal, carbono y los índices de diversidad fueron analizados mediante un análisis de varianza bajo un diseño completamente al azar con los tres tipos de bosques como tratamientos y nueve repeticiones. Se probaron los supuestos de normalidad, homocedasticidad e independencia mediante las pruebas de Shapiro-Wilks modificado y Levene y análisis gráfico de los errores. Se realizaron pruebas de LSD de Fisher para detectar diferencias entre pares de tipos de bosques $(\mathrm{p}<0,05)$. Todos los análisis se desarrollaron con el software Infostat (DI RIENZO et al., 2014).

\section{Resultados y discusión}

Se identificaron 22 familias, 26 géneros y 27 especies de árboles con dap $\geq 10 \mathrm{~cm}$ en los tres tipos de bosques en un esfuerzo de muestreo total de 0,405 ha. La riqueza encontrada en los bosques de estudio señala la importancia de la altitud, tal como lo afirman otros autores. Lozano et al. (2010) encontraron 412 especies de plantas herbáceas, arbustos y árboles, pertenecientes a 185 géneros y 75 familias en bosques de páramo del Parque Nacional Podocarpus en Loja, Ecuador. Entretanto, Peyre (2015) afirma que los páramos andinos poseen más de 3400 especies de plantas vasculares. Según Rangel (2002), las especies restringidas al ambiente paramuno son 405 y las especies encontradas con dap $\geq 9,9 \mathrm{~cm}$ en los bosques andinos del área de influencia del Páramo de Anaime representan el 7,4\% del total. 
No se detectaron diferencias significativas $(\mathrm{p}>0,05)$ en la abundancia entre los tres tipos de bosques: $309 \pm 37,243 \pm 35,210 \pm 33$ individuos/ha en bosque maduro, achaparrado y subpáramo, respectivamente (Figura 2). Solo se encontró una especie y un género comunes para los tres tipos de bosques: Weinmannia auriculata D. Don (encenillo; Cunoniaceae) y Miconia spp. (nigüito; Melastomataceae) con $430 \pm 50$ y $291 \pm 41$ individuos/ha, respectivamente (Figura 2). Montenegro y Vargas (2008) encontraron en la Reserva Forestal de Cogua (Cundimarca, Colombia) algunas de los géneros presentes en este estudio, tal como Tibouchina, Miconia, Weinmannia, Hedyosmum, Clethra, Myrsine y Oreopanax. La familia Cunoniaceae presentó la especie con mayor abundancia (Weinmannia auriculata), con el 31, 18 y 16\% del total del inventario forestal para los bosques achaparrados, maduro y subpáramo, respectivamente. La mayor dominancia en los bosques achaparrados fue alcanzada por Weinmannia auriculata con un valor de $56 \%$, seguido de Miconia spp. y Clusia sp. (Clusiaceae) con 23 y 12\%, respectivamente (Figura 3).

Figura 2 - Abundancia de árboles en tres tipos de bosque (total para subpáramo, maduro y achaparrado) en el Páramo de Anaime, Tolima, Colombia. Barras de error corresponden a error estándar. Anispp = Aniba sp.; Axispp = Axinaea sp.; Bacspp = Baccharis sp.; Bruboq = Brunellia boqueronensis; Budinc = Buddleja incana; Clespp = Clethra sp.; Cluspp = Clusia sp.; Corspp = Cordia sp.; Dipspp = Diplostephium sp.; Hedspp = Hedyosmun spp.; Hesfer = Hesperomeles ferruginea; Ilespp = Ilex sp.; Liaspp = Liabum sp.; Micspp = Miconia spp.; Mirspp = Myrcia sp.; Myrcor = Myrsine coriacea $;$ Myrdep = Myrsine dependens;

Ocospp = Ocotea sp.; Orespp = Oreopanax spp.; Palspp = Palicourea sp.; Perspp = Persea sp.; Quespp

$=$ Quercus humboldtii; Symspp = Symplocos spp.; Tibspp = Tibouchina sp.; Trisec $=$ Tristerix secundus; Vibana $=$ Viburnum anabaptista; Weiaur $=$ Weinmannia auriculata.

Figure 2 - Abundance of trees in three types of forests (total for subpáramo, mature and stumpy forests) in Páramo de Anaime, Tolima, Colombia. Bars of error correspond to standard error. Anispp = Aniba sp.; Axispp = Axinaea sp.; Bacspp = Baccharis sp.; Bruboq $=$ Brunellia boqueronensis; Budinc $=$ Buddleja incana; Clespp = Clethra sp.; Cluspp = Clusia sp.; Corspp = Cordia sp.; Dipspp = Diplostephium sp.; Hedspp = Hedyosmun spp.; Hesfer = Hesperomeles ferruginea; Ilespp = Ilex sp.; Liaspp = Liabum sp.; Micspp = Miconia spp.; Mirspp = Myrcia sp.; Myrcor = Myrsine coriacea $;$ Myrdep = Myrsine dependens; Ocospp = Ocotea sp.; Orespp = Oreopanax spp.; Palspp = Palicourea sp.; Perspp = Persea sp.; Quespp = Quercus humboldtii; Symspp = Symplocos spp.; Tibspp = Tibouchina sp.; Trisec $=$ Tristerix secundus; Vibana $=$ Viburnum anabaptista $;$ Weiaur $=$ Weinmannia auriculata .

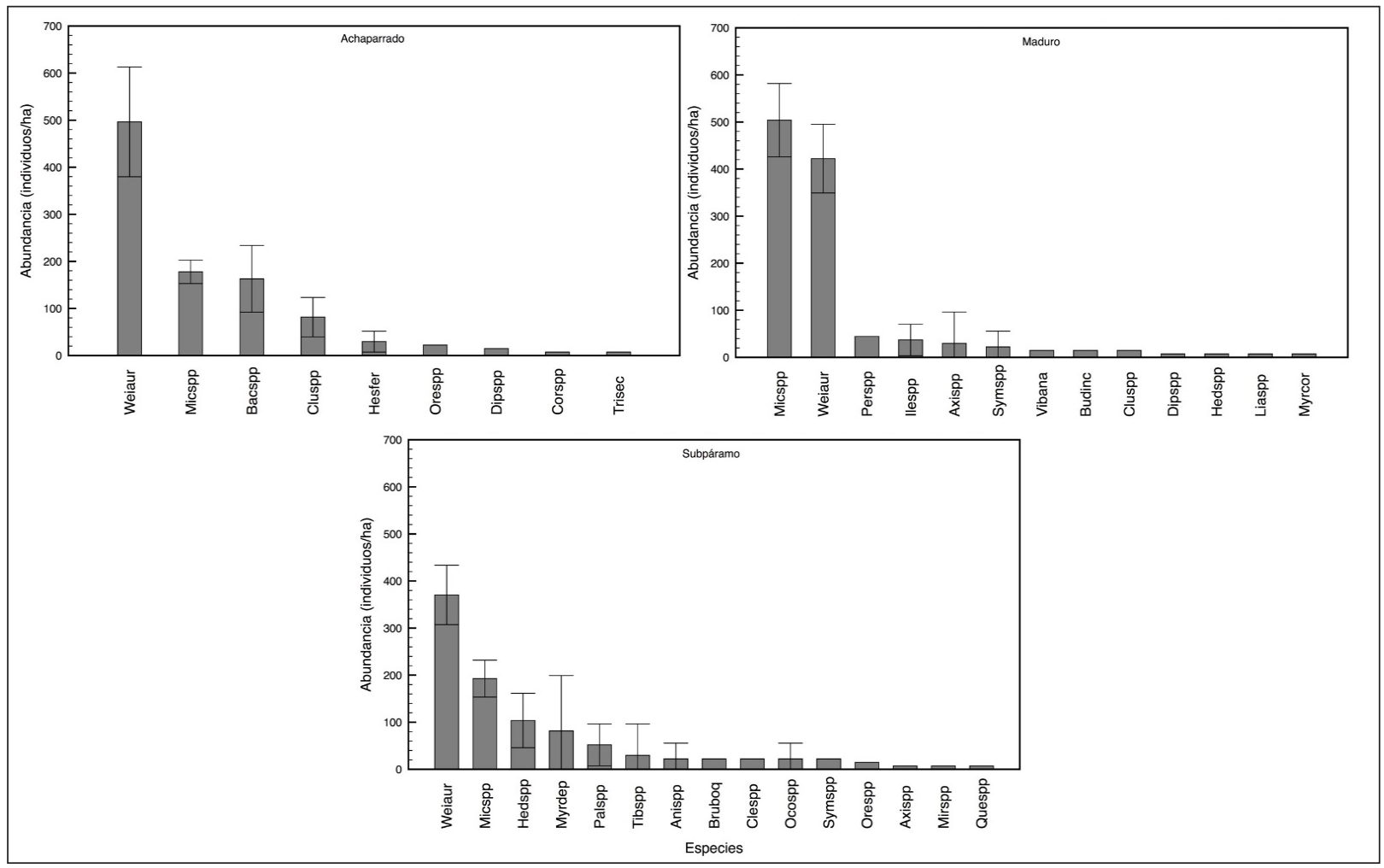

Ci. Fl., Santa Maria, v. 29, n. 1, p. 157-168, jan./mar., 2019 
Figura 3 - Dominancia absoluta de árboles en tres tipos de bosque (total para subpáramo, maduro y achaparrado) en el Páramo de Anaime, Tolima, Colombia. Barras de error corresponden a error estándar. Anispp = Aniba sp.; Axispp = Axinaea sp.; Bacspp = Baccharis sp.; Bruboq = Brunellia boqueronensis; Budinc $=$ Buddleja incana; Clespp $=$ Clethra $\mathbf{s p . ;}$ Cluspp = Clusia $\mathbf{s p . ;}$ Corspp $=$ Cordia sp.; Dipspp = Diplostephium sp.; Hedspp = Hedyosmun spp.; Hesfer = Hesperomeles ferruginea; Ilespp = Ilex sp.; Liaspp = Liabum sp.; Micspp = Miconia $\mathbf{s p p . ; ~ M i r s p p ~ = ~ M y r c i a ~} \mathbf{s p . ; ~ M y r c o r ~ = ~ M y r s i n e ~ c o r i a c e a ; ~}$ Myrdep = Myrsine dependens; Ocospp = Ocotea $\mathbf{s p . ;}$ Orespp = Oreopanax $\mathbf{s p p} . ;$ Palspp = Palicourea $\mathbf{s p . ;}$ Perspp = Persea $\mathbf{s p}$; Quespp = Quercus humboldtii; Symspp = Symplocos spp.; Tibspp = Tibouchina $\mathbf{s p . ;}$

Trisec $=$ Tristerix secundus; Vibana $=$ Viburnum anabaptista; Weiaur $=$ Weinmannia auriculata.

Figure 3 - Absolute dominance of trees in three types of forests (total for subpáramo, mature and stumpy forests) in Páramo de Anaime, Tolima, Colombia. Bars of error correspond to standard error. Anispp = Aniba sp.; Axispp = Axinaea sp.; Bacspp = Baccharis sp.; Bruboq = Brunellia boqueronensis; Budinc = Buddleja incana; Clespp = Clethra sp.; Cluspp = Clusia sp.; Corspp = Cordia sp.; Dipspp = Diplostephium sp.; Hedspp = Hedyosmun spp.; Hesfer = Hesperomeles ferruginea Ilespp = Ilex sp.; Liaspp = Liabum sp.; Micspp = Miconia spp.; Mirspp = Myrcia sp.; Myrcor = Myrsine coriacea; Myrdep = Myrsine dependens; Ocospp = Ocotea sp.; Orespp = Oreopanax spp.; Palspp = Palicourea sp.; Perspp = Persea sp.; Quespp =Quercus humboldtii; Symspp = Symplocos spp.; Tibspp = Tibouchina sp.; Trisec = Tristerix secundus; Vibana = Viburnum anabaptista; Weiaur $=$ Weinmannia auriculata .

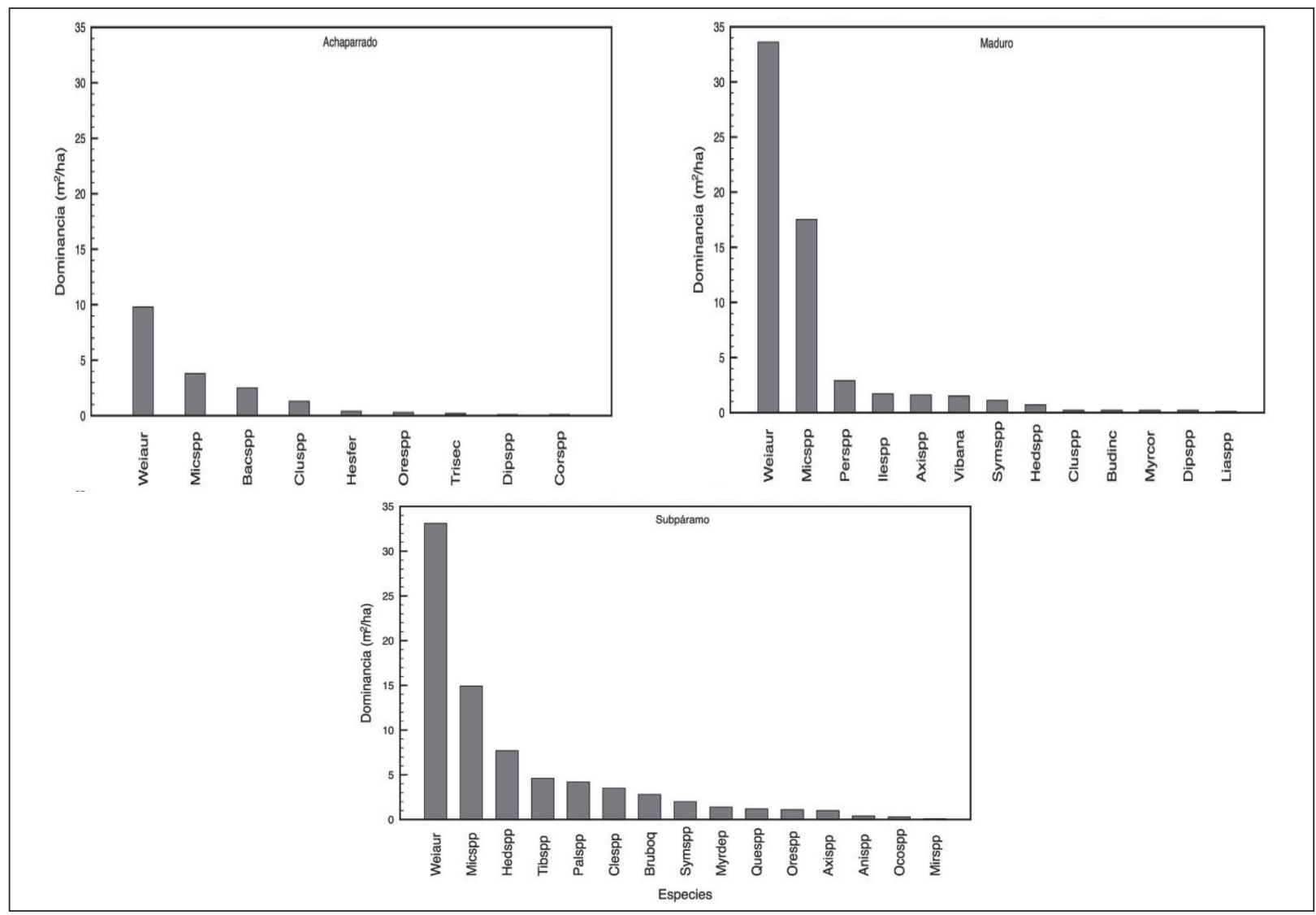

Las especies con mayor IVI en estos bosques fueron Weinmannia auriculata, Miconia spp. y Baccharis sp. (Asteraceae) con valores de 39, 25 y 17\%, respectivamente. Baccharis sp. en achaparrado, Hedyosmun spp. en subpáramo e Ilex sp. en bosque maduro también presentan una alta importancia ecológica, con un IVI de 17, 10 y 4\%, respectivamente (Figura 4). Los bosques estudiados presentaron una alta abundancia de epífitas como musgos, helechos, orquídeas y bromelias; sin embargo, no se encontraron lianas ni otros bejucos. Algunas de las especies más importantes, desde el punto ecológico, son endémicas de estas zonas de vida, tal como Brunellia boqueronensis y Quercus humboldtii, lo cual 
incrementa la necesidad de su conservación. De la misma forma, Quercus humboldtii es una especie vulnerable (CÁRDENAS y SALINAS, 2006).

La riqueza de especies arbóreas fue diferente entre tipos de bosque, en donde los del subpáramo y los maduros presentaron una mayor riqueza que los achaparrados (15 vs 13 vs 9 especies en 1350 $\mathrm{m}^{2}$ de área en esfuerzo de muestreo, respectivamente). Los índices de diversidad alfa son claves para estos análisis. De la misma forma, los tres índices de diversidad arrojaron diferencias estadísticas ( $\mathrm{p}$ $<0,05$ ) entre tipos de bosques (Tabla 1). De acuerdo a los índices de Margalef y Shannon-Wiener, la diversidad de los bosques del subpáramo fue significativamente superior $(\mathrm{p}<0,05)$ a los maduros; mientras que los bosques achaparrados se asemejan estadísticamente $(\mathrm{p}>0,05)$ a los otros dos tipos (Tabla 1). Los bosques maduros fueron más semejantes a los otros dos, lo que adiciona otra cualidad a estos ecosistemas con miras a conservación. Contrariamente, el índice de Simpson mostró que los del subpáramo son igualmente diversos estadísticamente $(\mathrm{p}>0,05)$ que los achaparrados pero significativamente $(\mathrm{p}<0,05)$ superiores a los maduros. De acuerdo a este índice, el bosque maduro está dominado por pocas especies, tal como las especies pertenecientes a los géneros Weinmannia y Miconia; mientras que los bosques del subpáramo presentaron una mayor equidad. Los bosques de la RNSA presentaron una alta diversidad beta por tener pocas especies en común (Tabla 2), en donde el bosque maduro es más semejante a los dos restantes; mientras que el achaparrado y subpáramo tienen pocas especies comunes (Tabla 2). Aunque este estudio solo incluyó plantas leñosas perennes, en el bosque de niebla del Parque Nacional Natural Chingaza (Colombia), Duque y Ríos (2011) encontraron un índice de uniformidad promedio de 0,67 en plantas vasculares.

Tabla 1 - Índices de diversidad alfa en tres tipos de bosque en el Páramo de Anaime, Tolima, Colombia.

Table 1 - Indexes of alpha diversity in three types of forests in the Páramo de Anaime, Tolima, Colombia.

\begin{tabular}{lccc}
\hline \multirow{2}{*}{ Variable } & \multicolumn{3}{c}{ Tipo de bosque } \\
\cline { 2 - 4 } & Achaparrado & Maduro & Subpáramo \\
\hline Riqueza de especies & 9 & 13 & 15 \\
Índice de Margalef & $1,2 \pm 0,2 \mathrm{ab}$ & $0,9 \pm 0,2 \mathrm{~b}$ & $1,4 \pm 0,1 \mathrm{a}$ \\
Índice de Simpson & $0,4 \pm 0,1 \mathrm{ab}$ & $0,5 \pm 0,0 \mathrm{a}$ & $0,3 \pm 0,0 \mathrm{~b}$ \\
Índice de Shannon- Wiener & $1,1 \pm 0,1 \mathrm{ab}$ & $1,0 \pm 0,1 \mathrm{~b}$ & $1,3 \pm 0,1 \mathrm{a}$ \\
\hline
\end{tabular}

Los valores corresponden a media \pm error estándar. Letras diferentes indican diferencias estadísticas entre tipos de bosques $(\mathrm{p}<0,05)$.

Se detectaron diferencias estadísticas $(\mathrm{p}<0,05)$ en la altura total y el dap de los árboles entre los tres tipos de bosques (Tabla 3). En los del subpáramo se detectaron los árboles de mayor tamaño, representado en estas dos variables dasométricas, que en los bosques maduros y achaparrados. Esto se ve reflejado en el área basal $(\mathrm{p}<0,05)$, la cual es $32,3 \%$ superior en el subpáramo que en los bosques achaparrados, respectivamente (Tabla 2). 
Figura 4 - Índice de valor de importancia (IVI) de árboles en tres tipos de bosque (total para subpáramo, maduro y achaparrado) en el Páramo de Anaime, Tolima, Colombia. Anispp = Aniba sp.; Axispp = Axinaea $\mathbf{s p} . ;$ Bacspp $=$ Baccharis $\mathbf{s p} . ;$ Bruboq $=$ Brunellia boqueronensis $;$ Budinc $=$ Buddleja incana $;$ Clespp = Clethra $\mathbf{s p} . ;$ Cluspp = Clusia $\mathbf{s p} . ;$ Corspp = Cordia $\mathbf{s p} . ;$ Dipspp = Diplostephium $\mathbf{s p} . ;$ Hedspp = Hedyosmun spp.; Hesfer = Hesperomeles ferruginea $;$ Ilespp = Ilex sp.; Liaspp = Liabum sp.; Micspp = Miconia spp.; Mirspp = Myrcia $\mathbf{s p . ;}$ Myrcor = Myrsine coriacea $;$ Myrdep = Myrsine dependens;

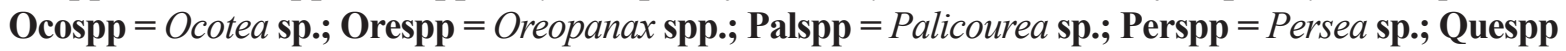

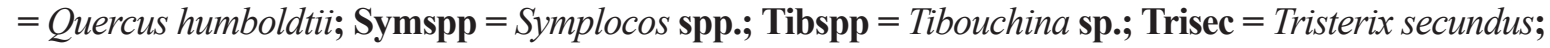
Vibana $=$ Viburnum anabaptista $;$ Weiaur $=$ Weinmannia auriculata .

Figure 4 - Importance value index of trees in three types of forests (total for subpáramo, mature and stumpy forests) in Páramo de Anaime, Tolima, Colombia. Anispp = Aniba sp.; Axispp = Axinaea sp.; Bacspp = Baccharis sp.; Bruboq = Brunellia boqueronensis; Budinc $=$ Buddleja incana $;$ Clespp $=$ Clethra sp.; Cluspp =Clusia sp.; Corspp = Cordia sp.; Dipspp = Diplostephium sp.; Hedspp = Hedyosmun spp.; Hesfer = Hesperomeles ferruginea $;$ Ilespp = Ilex sp.; Liaspp = Liabum sp.; Micspp = Miconia spp.; Mirspp = Myrcia sp.; Myrcor=Myrsine coriacea $;$ Myrdep = Myrsine dependens; Ocospp = Ocotea sp.; Orespp = Oreopanax spp.; Palspp = Palicourea sp.; Perspp = Persea sp.; Quespp = Quercus humboldtii; Symspp = Symplocos spp.; Tibspp = Tibouchina $\mathrm{sp} . ;$ Trisec $=$ Tristerix secundus; Vibana $=$ Viburnum anabaptista , Weiaur $=$ Weinmannia auriculata .

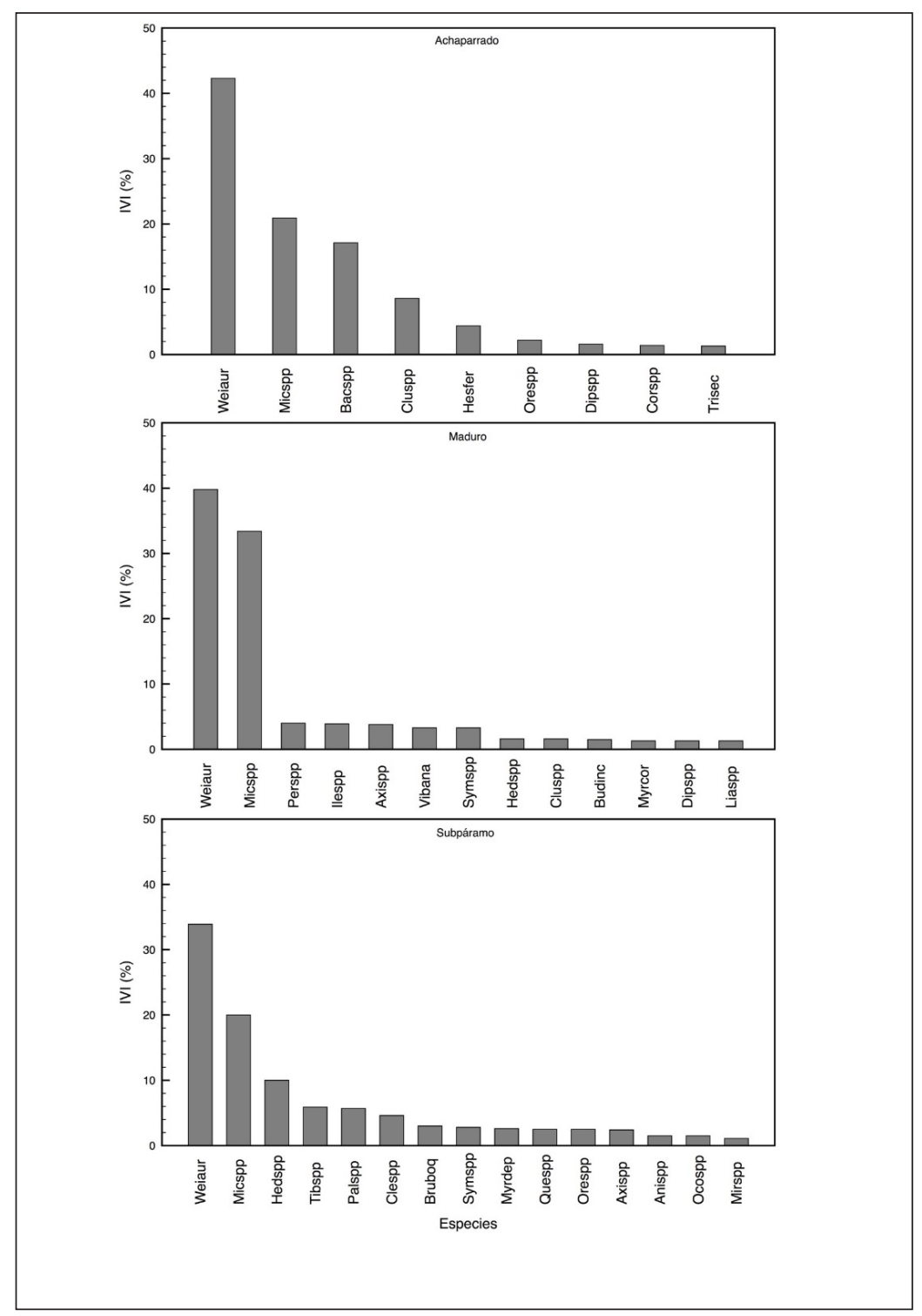


Tabla 2 - Índices de diversidad beta en tres tipos de bosque en el Páramo de Anaime, Tolima, Colombia.

Table 2 - Indexes of beta diversity of trees in three type of forests in Páramo de Anaime, Tolima, Colombia.

\begin{tabular}{lccc}
\hline \multirow{2}{*}{ Índice } & \multicolumn{3}{c}{ Tipos de bosque } \\
\cline { 2 - 4 } & Achaparrado-maduro & Achaparrado-subpáramo & Maduro-subpáramo \\
\hline Jaccard & 0,22 & 0,14 & 0,22 \\
Sørensen & 0,36 & 0,25 & 0,36 \\
Complementariedad & 0,78 & 0,86 & 0,78 \\
\hline
\end{tabular}

Los bosques del subpáramo y los maduros almacenaron estadísticamente $(\mathrm{p}<0,05)$ más carbono que los achaparrados $(144,9 \pm 33,2$ y $110,5 \pm 17,3$ vs $32,2 \pm 5,5 \mathrm{Mg} / \mathrm{ha}$, respectivamente; Tabla 3). El carbono en los bosques maduro y achaparrado (128 Mg/ha) está en el rango reportado por Phillips et al. (2011) para bosques andinos colombianos (48,1 a 129,4 Mg/ha) y por Köhl et al. (2015) en bosques de América del Sur (122 Mg C ha). En Ecuador, Fehse et al. (2002) estimaron entre 88 y $183 \mathrm{Mg} / \mathrm{ha}$ el carbono almacenado en bosques secundarios de Alnus y Polylepis de 30 y 45 años de edad. La capacidad de captura de carbono se reduce al incrementarse la altitud. Sin embargo, a pesar de las bajas temperaturas de la zona, los bosques maduros y del subpáramo retienen gran cantidad de carbono en su biomasa aérea; adicionalmente, se encuentran amenazados (ETTER et al., 2006) porque incrementos en las temperaturas globales harían ascender algunas especies y cambiar su composición florística. Los bosques más importantes para el almacenamiento de carbono y preservación con miras a mitigación del cambio climático son los maduros, principalmente debido a su extensión en la RNSA, ya que en toda el área conservan entre tres y siete veces más que los otros (2,2 $\mathrm{Tg} \mathrm{CO}_{2}$; Tabla 3).

Tabla 3 - Características dasométricas, carbono almacenado en biomasa aérea y emisiones evitadas en bosques nativos en la Reserva Natural Semillas de Agua, Páramo de Anaime, Tolima, Colombia.

Table 3 - Dasometric characteristics, carbon storage in aboveground biomass and avoided emissions in native forests in Reserva Natural Semillas de Agua, Páramo de Anaime, Tolima, Colombia.

\begin{tabular}{|c|c|c|c|c|c|c|c|}
\hline \multirow{3}{*}{ Tipo de bosque } & \multirow{3}{*}{$\operatorname{dap}(\mathrm{cm})$} & \multirow{3}{*}{ Altura total (m) } & \multirow{3}{*}{$\begin{array}{c}\text { Área basal } \\
\left(\mathbf{m}^{2} / \mathbf{h a}\right)\end{array}$} & \multirow{3}{*}{$\begin{array}{c}\text { Carbono } \\
\text { (Mg/ha) }\end{array}$} & \multirow{3}{*}{$\begin{array}{c}\text { Carbono } \\
\text { total aéreo } \\
\left(\mathrm{Tg}^{\circ} \mathrm{CO}_{2^{\circ}}\right)\end{array}$} & \multicolumn{2}{|c|}{ Emisión Total (Gg CO$\left.)_{2}\right)$} \\
\hline & & & & & & \multicolumn{2}{|c|}{ Tasas de deforestación (\%/año) } \\
\hline & & & & & & 0,28 & 0,78 \\
\hline Achaparrado & $14,5 \pm 0,4 \mathrm{c}$ & $10,0 \pm 0,3 \mathrm{c}$ & $18,5 \pm 2,9 b$ & $32,2 \mathrm{~b}$ & 0,31 & 16,7 & 44,4 \\
\hline Maduro & $23,6 \pm 0,9 \mathrm{~b}$ & $17,6 \pm 0,5 \mathrm{~b}$ & $61,3 \pm 9,4 \mathrm{a}$ & $110,5 \mathrm{a}$ & 2,18 & 118,8 & 315,7 \\
\hline Subpáramo & $27,4 \pm 1,4 \mathrm{a}$ & $19,6 \pm 0,9 \mathrm{a}$ & $78,3 \pm 17,5 \mathrm{a}$ & $144,9 \mathrm{a}$ & 0,69 & 37,4 & 99,5 \\
\hline Total & & & & & 3,17 & 172,9 & 459,6 \\
\hline
\end{tabular}

$1 \mathrm{Mg}=10^{6} \mathrm{~g} ; 1 \mathrm{Gg}=10^{9} \mathrm{~g} ; 1 \mathrm{Tg}=10^{12} \mathrm{~g}$. Los valores corresponden a media \pm error estándar. Letras diferentes indican diferencias estadísticas entre tipos de bosques $(\mathrm{p}<0,05)$. Área de tipos de bosque $=2589$ ha para achaparrado, 5370 ha para maduro y 1291 ha para los del subpáramo. 
La RNSA almacena en total 3,2 $\mathrm{Tg} \mathrm{CO}_{2}$, de los cuales se podrían emitir entre 119 y $316 \mathrm{Gg}$ $\mathrm{CO}_{2}$ en 20 años (Tabla 3) si no se establece un proyecto que propenda por su conservación, tal como los REDD+ (ETTER et al., 2006). Los bosques húmedos premontanos (bh-PM) del país retienen $388 \mathrm{Tg} \mathrm{CO}_{2}$ (PHILLIPS et al., 2011). Los bosques achaparrados serían los menos relevantes para su conservación, en términos de reducción de emisión de GEI, ya que se conservarían solo 17 a $44 \mathrm{Mg} \mathrm{CO}_{2}$ durante el mismo periodo (Tabla 3). Al cabo de 20 años, si no se tomaran acciones para detener los procesos de deforestación, estos bosques llegarían a almacenar entre 2,7 y 3,0 $\mathrm{Tg} \mathrm{CO}_{2}$, lo cual significa una emisión de 0,2-0,5 $\mathrm{Tg} \mathrm{CO}_{2}$ a la atmósfera (Tabla 3). La estructura y diversidad de estos bosques se está viendo amenazada por la alta importancia ecológica de Weinmannia auriculata, la cual es una especie que está recibiendo una alta presión de explotación para leña (DUQUE y RÍOS, 2011).

\section{Conclusiones}

Los bosques altoandinos de la Reserva Natural Semillas de Agua (RNSA) son un albergue importante de diversidad de especies arbóreas, principalmente algunas que son endémicas de la zona. Weinmannia auriculata, Miconia spp. y Baccharis sp. son las especies más importantes desde el punto de vista ecológico en los bosques del Páramo de Anaime. Los bosques del subpáramo y maduros fueron los más diversos en árboles; sin embargo, este último presenta mayor similaridad con los dos restantes, resultado obvio de la localización altitudinal.

Aunque los bosques estudiados presentaron una abundancia similar, se observaron valores de área basal y de carbono muy diferente entre ellas, demostrando las diferencias en los tamaños de los individuos, en términos de dap y altura total. Weinmannia auriculata fue la especie más importante ecológicamente y en su contenido de carbono, lo cual implica la necesidad de manejarla sosteniblemente e investigar más en profundidad su ecología.

Los bosques del subpáramo y maduros almacenan más carbono en biomasa aérea. La RNSA tienen almacenado en total 3,2 $\mathrm{Tg} \mathrm{CO}_{2}$ en biomasa aérea, la que está en peligro en caso de mantenerse o incrementarse las tasas de deforestación en la zona, pudiendo causar una emisión neta de 9 a $23 \mathrm{Gg} \mathrm{CO}_{2} \mathrm{e} /$ año. Estas serían las reducciones de emisiones que serían atribuidas a un proyecto, tal como REDD+y las que podrían ser comercializadas para fomentar proyectos de conservación y manejo sostenible.

\section{Agradecimientos}

Los autores desean agradecer a Corporación Semillas de Agua por su invaluable apoyo asistencial en logística y consecución de parcelas de productores participantes. De la misma forma, agradecemos a la Alcaldía de Cajamarca, Tolima por su apoyo financiero. A todos ellos, nuestro más sincero reconocimiento.

\section{Referencias}

ANDRADE, H. J.; ESPINOSA, E. L.; MORENO, H. A. Impact of grazing in soil organic storage carbon in high lands of Anaime, Tolima, Colombia. Zootecnia Tropical, Maracay, v. 32, n. 1, p. 7-21, 2014.

ANGELSEN, A. et al. Reducción de emisiones de la deforestación y la degradación de bosques (REDD): Reporte de evaluación de opciones. Meridian Institute. Washington, D.C., USA, 2009. 108 p.

ASORED - Asociación red Colombiana de Reservas Naturales de la Sociedad Civil. Ficha de Caracterización Reserva Natural Semillas de Agua. Ibagué, 2006, 37 p.

BISHOP, J.; LANDELL-MILLS, N. Los servicios ambientales de los bosques. In: PAGIOLA, S.; BISHOP, J.; N LANDELL-MILLS, E. (Comp.). La venta de servicios ambientales forestales. Secretaría de Medio Ambiente y Recursos Naturales; Instituto Nacional de Ecología. México, 2003, p. 47-74.

BROWN, S.; LUGO, A. E. The storage and production of organic matter in tropical forests and their role in the global carbon cycle. Biotropica, Washington, v. 14, n. 3, p. 161-187, 1982. 
CABRERA, E. et al. Memoria técnica de la cuantificación de la deforestación histórica nacional - escalas gruesa y fina. Bogotá: Instituto de Hidrología, Meteorología, y Estudios Ambientales IDEAM, 2011, $106 \mathrm{p}$.

CÁRDENAS, D.; SALINAS, N. R. Libro rojo de plantas de Colombia. Especies maderables amenazadas Parte I. Bogotá, Colombia: Ministerio de Ambiente, Vivienda y Desarrollo Territorial, 2006, 169 p.

COOMES, O. et al. The Fate of the Tropical Forest: Carbon or Cattle? Ecological Economics, Amsterdam, v. 65 , n. 2, p. 207-212, abr. 2008.

CORPORACIÓN SEMILLAS DE AGUA. Plan de manejo integrado del páramo de Anaime y Chili. Cali, 2009, 45 p.

CORPORACIÓN SEMILLAS DE AGUA \& WWF COLOMBIA. Cálculo tasas de deforestación construcción iniciativa REDD+ Anaime y Chili. Ibagué, Colombia, 2011, 2 p.

DI RIENZO, J. A. et al. InfoStat versión 2014. Grupo InfoStat, Facultad de Ciencias Agropecuarias, Universidad Nacional de Córdoba, Argentina. Disponible: http://www.infostat.com.ar. Acceso: 2 feb. 2014.

DUQUE, J.; RÍOS, M. Caracterización y especialización de las plantas vasculares presentes en un área con degradación de cobertura vegetal en los ecosistemas páramo húmedo y bosque de niebla, propios del Parque Nacional Natural Chingaza. Sumapaz, Fusagasugá, n. 1, p. 57-77, 2011.

ETTER, A. et al. Regional patterns of agricultural land use and deforestation in Colombia. Agriculture, Ecosystems and Environment, Amsterdam, v. 114, n. 2-4, p. 369-386, 2006.

FAO - Food and Agriculture Organization. Global Forest Resources Assessment 2010. Forestry Paper 163. Rome. IT, FAO, 2010, $340 \mathrm{p}$.

FEHSE, J. et al. High altitude tropical secondary forests: a competitive carbon sink? Forest Ecology and Management, Amsterdam, v. 163, n. 1-3, p. 9-25, 2002.

GILLESPIE, A. J. R.; BROWN, S.; LUGO, A. E. Tropical forest biomass estimation from truncated stand tables. Forest Ecology and Management, Amsterdam, v. 48, n. 1-2, p. 69-87, 1992.

GÓMEZ, R. et al. Informe de seguimiento a la financiación de REDD+ 2009-2012. Bogotá, Fundación Natura, 2013, $35 \mathrm{p}$.

HOLDRIDGE, L. R. Life Zone Ecology. Tropical Science Center. San José, Costa Rica. 1a. ed. San José, Costa Rica: IICA, 1967.

IPCC - Intergovernmental Panel on Climate Change. IPCC Guidelines for National Greenhouse Gas Inventories. Intergovernmental Panel on Climate Change National Greenhouse Gas Inventories Programme. Disponible: http://www.ipcc-nggip.iges.or.jp/public/2006gl/. Acceso: 1 marzo 2006.

KESSLER, M. The Elevational Gradient of Andean Plant Endemism: Varying Influences of TaxonSpecific Traits and Topography at Different Taxonomic Levels. Journal of Biogeography, Washington, v. 29, n. 9, p. 1159-1165, 2002.

KÖHL, M. et al. Changes in forest production, biomass and carbon: Results from the 2015 UN FAO Global Forest Resource Assessment. Forest Ecology and Management, Amsterdam, v. 352, p. 21-34, sept. 2015.

LOZANO, P.; KÜPPERS, M.; BUSSMANN, R. W. Plant diversity of paramo and andean elfin forest in Podocarpus National Park - Loja, Ecuador. Arnaldoa, Trujillo, v. 17, n. 2, p. 193-202, jul-dic. 2010.

LUGO, A.E.; BROWN, S. Tropical forests as sinks of atmospheric carbon. Forest Ecology and Management, Amsterdam, v. 54, n. 1-4, p. 239-255, nov. 1992.

MEDELLÍN, H. Valoración económica ambiental de páramos. La valoración económica como herramienta de Gestión Ambiental. Disponible: http://www.banrepcultural.org/blaavirtual/geografia/ congresoparamo/valoracion.pdf=. Acceso: 1 marzo 2011. 
MADS - Ministerio de Ambiente y Desarrollo Sostenible. Informe de Gestión al Congreso. Disponible: http://www.minambiente.gov.co/documentos/normativa/240712_informe_gestion_mads_original.pdf. Acceso: 1 marzo 2012.

MONTENEGRO, A. L.; VARGAS, O. Caracterización de bordes de bosque altoandino e implicaciones para la restauración ecológica en la Reserva Forestal de Cogua (Colombia). Revista de Biología Tropical, San José, v. 56, n. 3, p. 1543-1556, 2008.

PEYRE, G. Plant diversity and vegetation of the Andean Páramo. A thesis submitted for the degree of Doctor, University of Barcelona and Aarhus University, Barcelona, Spain, 2015, 82 p + appendixes.

PHILLIPS, J. F. et al. Estimación de las reservas potenciales de carbono almacenadas en la biomasa aérea en bosques naturales de Colombia. Instituto de Hidrología, Meteorología y Estudios AmbientalesIDEAM. Bogotá D.C., Colombia, 2011, 32 p.

RANGEL, O. Biodiversidad en la región del páramo: con especial referencia a Colombia. En: CONGRESO MUNDIAL DE PÁRAMOS, 13 al 18 de mayo, 2002. Paipa, Colombia, 2002, p. 168-200.

RANGEL-CHURIO, J. O.; PINTO-ZÁRATE, J. H. Colombian Páramo Vegetation Database (CPVD) - the database on high Andean páramovegetation in Colombia. Biodiversity \& Ecology, Hamburg, v. 4, p. 275-286, 2012.

RUDAS, G. et al. Biodiversidad y actividad humana: relaciones en ecosistemas de bosque subandino en Colombia. Instituto de Investigación de Recursos Biológicos Alexander von Humboldt. Bogotá D. C., Colombia, 2007. 128 p.

RÜGNITZ, M.; CHACÓN, M.; PORRO, R. Guía para la Determinación de Carbono en Pequeñas Propiedades Rurales. 1 ed Lima, Perú.: Centro Mundial Agroflorestal (ICRAF) / Consórcio Iniciativa Amazônica (IA), 2009, 79 p.

SALIMON, C. I. et al. Estimating state-wide biomass carbon stocks for a REDD plan in Acre, Brazil. Forest Ecology and Management, Amsterdam, v. 262, n. 3, p. 555-560, Ago. 2011.

SEGURA, M. et al. Almacenamiento y fijación de carbono en bosques de bajura de la zona atlántica de Costa Rica. Revista Forestal Centroamericana, Turrialba, v. 30, p. 23-28, abr-jun, 2000.

SEGURA, M. et al. Modelos alométricos para estimar biomasa aérea total de las especies dominantes en bosques altoandinos, Colombia. In: 4TO CONGRESO FORESTAL ARGENTINO Y LATINOAMERICANO, 2013, Iguazú, Argentina.

SPRACKLEN, D. V.; RIGHELATO, R. Tropical montane forests are a larger than expected global carbon store. Biogeosciences, Goettingen, v. 11, 2741-2754, 2014.

URBINA-CARDONA, J. N. Gradientes andinos en la diversidad y patrones de endemismo en anfibios y reptiles de Colombia: posibles respuestas al cambio climático. Revista Facultad de Ciencias Básicas, Bogotá, v. 7, n. 1, p. 74-91, 2011.

VÁSQUEZ, A.; BUITRAGO, A. C. EI gran libro de los páramos. Instituto de Investigación de Recursos Biológicos Alexander von Humboldt. Proyecto Páramo Andino. Bogotá, D.C, 2011. 